\title{
Cisto dentígero: diagnóstico e tratamento
}

\author{
Cisto dentígero: diagnosis and treatment \\ Cisto dentígero: diagnóstico y tratamiento \\ Rodolfo Pollo SOARES ${ }^{1}$ \\ Aline Reis STEFANINI ${ }^{2}$ \\ André Luis da Silva FABRIS ${ }^{3}$ \\ Paulo Henrique BORTOLUZZO 3 \\ Luciana Estevam SIMONATO ${ }^{4}$ \\ ${ }^{I}$ Acadêmico do Curso de Odontologia da Universidade Brasil, Campus Fernandópolis, 15600-000 Fernandópolis-SP, Brasi \\ ${ }^{2}$ Docente do Cursos de Medicina da Universidade Brasil, Campus Fernandópolis, 15600-000 Fernandópolis-SP, Brasil \\ ${ }^{3}$ Docente do Curso de Odontologia da Universidade Brasil, Campus Fernandópolis, 15600-000 Fernandópolis-SP, Brasil \\ ${ }^{4}$ Docente dos Cursos de Medicina e Odontologia da Universidade Brasil, Campus Fernandópolis, 15600-000 Fernandópolis-SP, Brasil
}

\begin{abstract}
Resumo
O cisto dentígero é um cisto odontogênico que é classificado como de desenvolvimento. Normalmente, está relacionado à coroa de um dente incluso, sendo um dos cistos odontogênicos mais frequentes nos ossos gnáticos. Na maioria das vezes é diagnosticado em pacientes entre a segunda e a terceira década de vida, com grande ocorrência em terceiros molares inferiores e caninos superiores. Clinicamente, apresenta evolução lenta, assintomática e pode causar discreta deformidade facial, deslocamento de dentes e alterações de estruturas na região. Radiograficamente, os cistos dentígeros são descritos como lesões radiolúcidas bem delimitadas e uniloculares. Na maioria dos casos, são observados em exames de rotina ou durante a pesquisa da causa da não erupção de um dente permanente. Apesar da singularidade clínica de cada caso, o prognóstico dessa lesão é favorável. O tratamento para o cisto dentígero pode ser a marsupialização em casos de lesões grandes, enucleação com exodontia do dente incluso ou preservação do elemento dental. Este trabalho visa apresentar um caso clínico de cisto dentígero em região posterior de mandíbula, abordando aspectos clínicos, imaginológicos, histopatológicos e terapêuticos, com a finalidade de familiarizar o cirurgião dentista com tal lesão.
\end{abstract}

Descritores: Cisto; Cisto Dentígero; Diagnóstico Bucal.

\section{Abstract}

The dentigerous cyst is an odontogenic cyst that is classified as developmental cyst. It is usually related to the crown of an included tooth, being one of the most frequent odontogenic cysts in the gnatic bones. Most of the time it is diagnosed in patients between the second and third decade of life, with great occurrence in third molars and upper canines. Clinically, it presents slow evolution, asymptomatic and can cause discreet facial deformity, tooth dislocation and alterations of structures in the region. Radiographically, dentigerous cysts are described as well delimited and unilocular radiolucent lesions. Normally, they are seen in routine examinations or during the investigation of the cause of non-eruption of a permanent tooth. Despite the clinical singularity of each case, the prognosis of this lesion is favorable. The treatment for dentigerous cyst may be marsupialization in cases of large lesions, enucleation with exodontia of the included tooth or preservation of the dental element. This paper aims to present a case report of a dentigerous cyst in the mandible, posterior region, addressing clinical, imaging, histopathological and therapeutic aspects, in order to familiarize the dentist surgeon with such lesion.

Descriptors: Cyst; Dentiger Cyst; Oral Diagnosis.

\section{Resumen}

El quiste dentígero es un quiste odontogénico que se clasifica como de desarrollo. Normalmente, esta relacionada con la corona de un diente incluido, siendo uno de los quistes odontogénicos más frecuentes en los huesos gnáticos. La mayoría de las veces se diagnostica en pacientes entre la segunda y la tercera década de vida, con gran ocurrencia en terceros molares inferiores y caninos superiores. Clínicamente, presenta evolución lenta, asintomática y puede causar discreta deformidad facial, desplazamiento de dientes y cambios de estructuras en la región. Los cistos dentígeros se describen como lesiones radiolúcidas bien delimitadas y uniloculares. Normalmente, se observan en exámenes de rutina o durante la investigación de la causa de la no erupción de un diente permanente. A pesar de la singularidad clínica de cada caso, el pronóstico de esta lesión es favorable. El tratamiento para el quiste dentígero puede ser la marsupialización en casos de lesiones grandes, enucleación con exodoncia del diente incluido o preservación del elemento dental. Este trabajo pretende presentar un relato de caso clínico de quiste dentígero en mandíbula, región posterior, abordando aspectos clínicos, imaginológicos, histopatológicos y terapéuticos, con la finalidad de familiarizar al cirujano dentista con tal lesión.

Descriptores: Cisto; Quiste Dental; Diagnóstico Bucal.

\section{INTRODUÇÃO}

O cisto dentígero é uma lesão benigna, formada por epitélio odontogênico proveniente da coroa de um dente não erupcionado ${ }^{1}$. Sua etiologia ainda é incerta ${ }^{1}$ e é considerado o segundo cisto odontogênico mais frequente nos ossos gnáticos ${ }^{2-7}$. Clinicamente, os cistos dentígeros são, usualmente, assintomáticos $^{3}$, com crescimento lento e caracterizados pelo envolvimento da coroa e erupção retardada da unidade dental envolvida ${ }^{7,8}$. Vale ressaltar que as lesões de grande extensão estão normalmente relacionadas à expansão e erosão da cortical óssea, podendo assim causar uma parestesia no nervo alveolar inferior, quando presente em mandíbula9.

Radiograficamente, o cisto dentígero é observado como uma imagem radiolúcida unilocular bem delimitada envolta por um halo esclerótico, associada à coroa de um dente incluso ${ }^{4-6,10,11}$. Segundo Neville ${ }^{12}$ variações radiográficas são observadas, podendo classificar o cisto dentígero em central, lateral e circunferencial, de acordo com a relação existente entre a lesão e a coroa do dente incluso. Outras alterações radiográficas podem ocorrer, dependendo de cada caso, resultado em lesões com limites mal definidos e com aspecto multilocular, respectivamente, nos cistos infectados e nos cistos de grande dimensão ${ }^{13-15}$. Os cistos dentígeros possuem potencial para se tornar extremamente grandes e promover a expansão da cortical óssea ${ }^{6}$.

Histopatologicamente, o cisto dentígero consiste de uma parede fibrosa que pode conter 
quantidades variáveis de tecido mixóide e restos odontogênicos. Ele é circundado por um epitélio estratificado escamoso não-ceratinizado, que pode exibir ocasionalmente células mucosas, ciliadas e, raramente, células sebáceas ${ }^{6}$. Às vezes, o epitélio é do tipo reduzido do esmalte, com poucas camadas de células, com presença de corpúsculos hialinos. A cápsula fibrosa pode apresentar cordões de epitélio odontogênico e calcificações distróficas ${ }^{16.17}$.

$\mathrm{O}$ diagnóstico da lesão não deve ser feito unicamente sobre evidências radiográficas, mas também baseado nas evidências clínicas e no exame microscópico do espécime ${ }^{18}$, por causa da associação com várias outras lesões, tais como ameloblastomas unicísticos $^{19}$ e ceratocistos odontogênicos ${ }^{2}$, que também podem ocorrer nestes locais.

O método de tratamento padrão consiste na excisão completa do cisto com a remoção do dente associado $^{20}$. É a enucleação do cisto e extração do dente não irrompido esta modalidade de tratamento é empregada em cerca de $85 \%$ dos $\operatorname{casos}^{21,22}$. Nestes pacientes, o dente não irrompido é considerado sem maior utilidade à função mastigatória/ estética ou por falta de espaço no arco para irrompimento ${ }^{22}$.

Apesar da singularidade clínica de cada caso, este trabalho visa apresentar relato de cisto dentígero em mandíbula, região posterior, abordando aspectos clínicos, imaginológicos, histopatológicos e terapêuticos com a finalidade de familiarizar o cirurgião-dentista com tal lesão.

\section{CASO CLÍNICO}

Paciente A.F.G, 44 anos, gênero feminino, leucoderma, procedente de Fernandópolis (SP), compareceu ao Centro de Especialidades Odontológicas (CEO) de Fernandópolis encaminhado por cirurgião dentista de Unidade Básica de Saúde (UBS) em decorrência de lesão intraóssea observada em exame radiográfico de rotina.

Durante o exame físico extraoral não foi verificado nenhuma alteração digna de nota. No entanto, no exame físico intraoral percebeu-se que na região do dente 48 , que se apresentava em erupção, havia um discreto abaulamento. A mucosa que revestia a área apresentava-se normocrômica e íntegra. À palpação, a paciente não referia dor ou qualquer outro incomodo.

Após avaliação clínica detalhada, foram solicitados exames imaginológicos, sendo radiografia panorâmica e tomografia computorizada. $\mathrm{Na}$ radiografia panorâmica observou-se lesão radiolúcida bem delimitada por um halo radiopaco, envolvendo parcialmente o dente 48, que se encontrava semiincluso e impactado. A lesão intraóssea apresentava cerca de $2,5 \mathrm{~cm}$ de diâmetro e envolvia área do terceiro molar inferior direito até o ângulo da mandíbula do lado direito, sendo que as raízes da unidade envolvida se apresentavam divergentes e com processo de reabsorção (Figura 1). A tomografia computadorizada reforçou os achados radiográficos demostrando, também, a proximidade da lesão com o canal da mandíbula do lado direito (Figura 2).

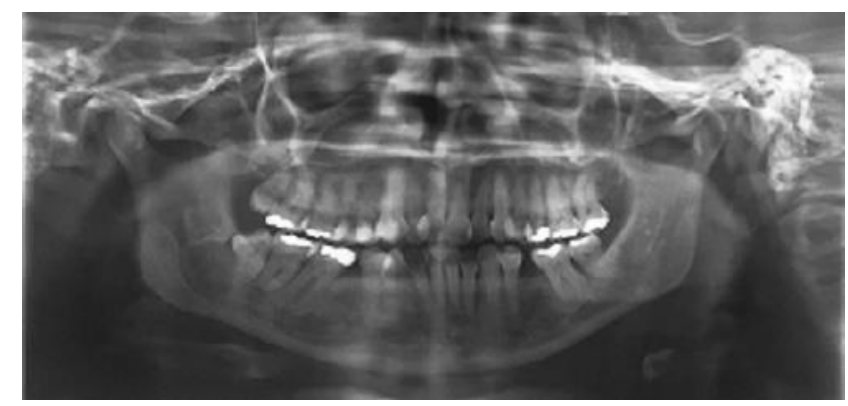

Figura 1: Exame radiográfico inicial.

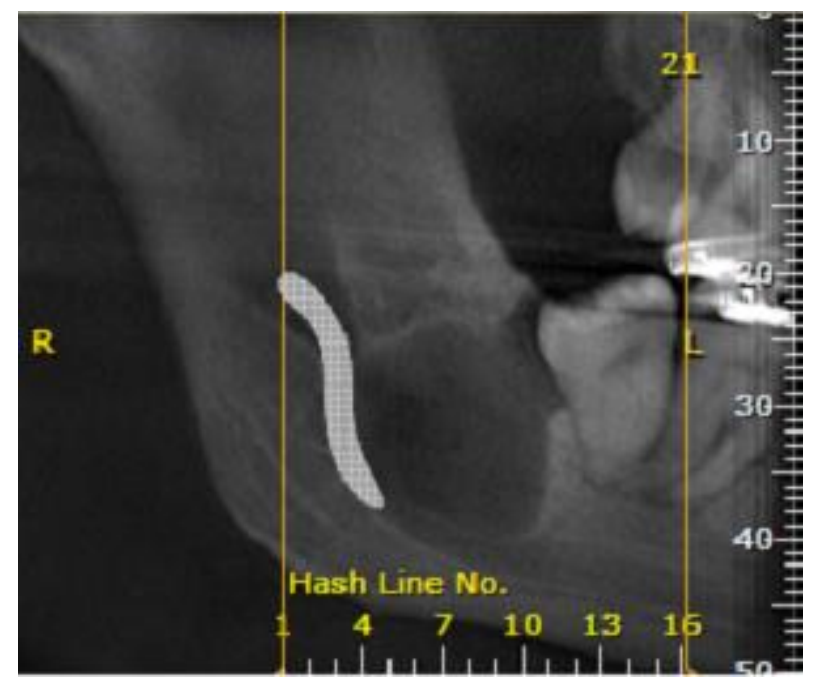

Figura 2: Exame tomográfico inicial.

Mediante os achados clínicos e radiográficos, o diagnóstico sugestivo foi de cisto dentígero. Dessa forma, a conduta inicial foi punção aspirativa do conteúdo da lesão que reforçou a hipótese diagnóstica (Figura 3). Em seguida, foi realizada enucleação cirúrgica da lesão com anestesia local e o material coletado foi encaminhado para análise em serviço especializado (Figura 4).

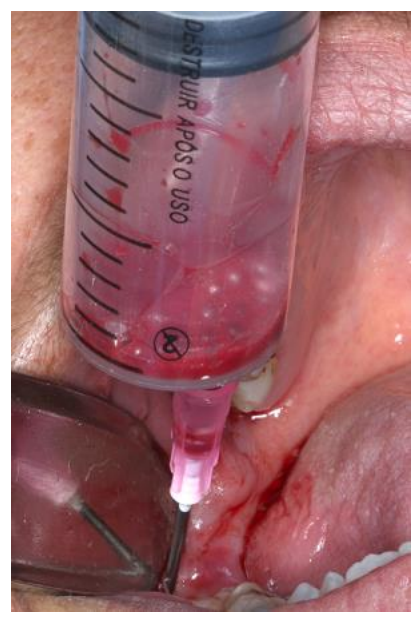

Figura 3: Punção aspirativa. 


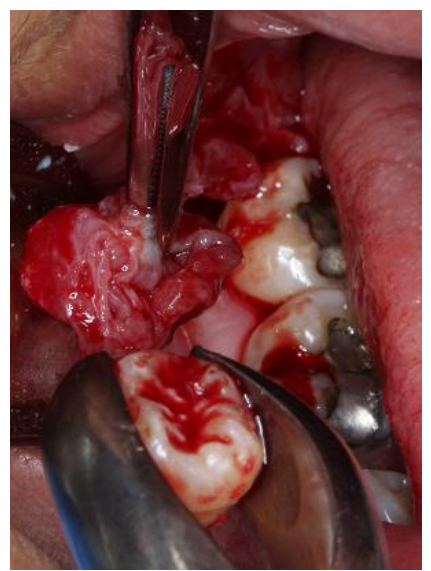

Figura 4: Enucleação cirúrgica.

Os achados histopatológicos determinaram cisto dentígero. O cisto apresentava-se envolvido por uma cápsula densa, o que facilitou sua total remoção, excisão e preservação do nervo alveolar inferior e toda a espessura do ângulo da mandíbula (Figura 5). Os elementos dentários 47 e 48 foram extraídos juntamente com a lesão e os outros elementos foram mantidos e conservados.

Durante o período de 06 meses, a paciente foi acompanhada e não houve qualquer irregularidade no pós-operatório, relacionado à parestesia, ou qualquer recidiva da lesão. Os elementos próximos à lesão apresentaram resultados positivos no teste de vitalidade. A radiografia panorâmica, realizada 06 meses após a cirurgia, confirmou a substituição do tecido lesado, neoformação de tecido ósseo na região (Figura 6). A paciente ainda está sendo acompanhada, sem sinais clínicos e radiográficos de recidiva da lesão.

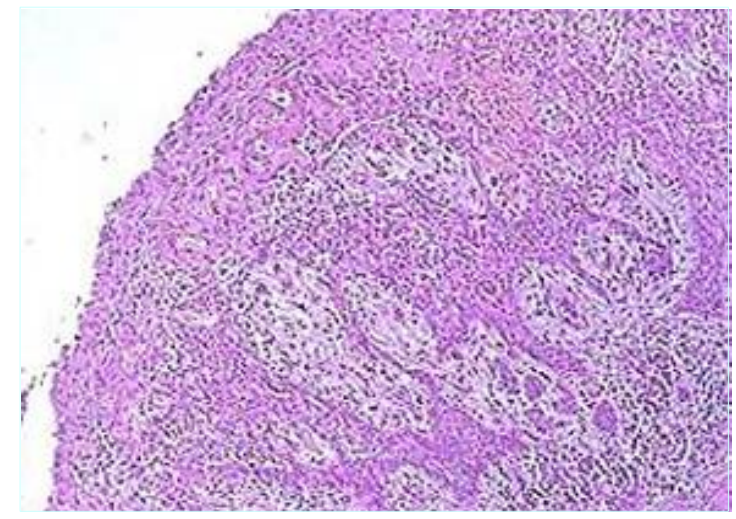

Figura 5: Exame histopatológico. H.E. 100x.

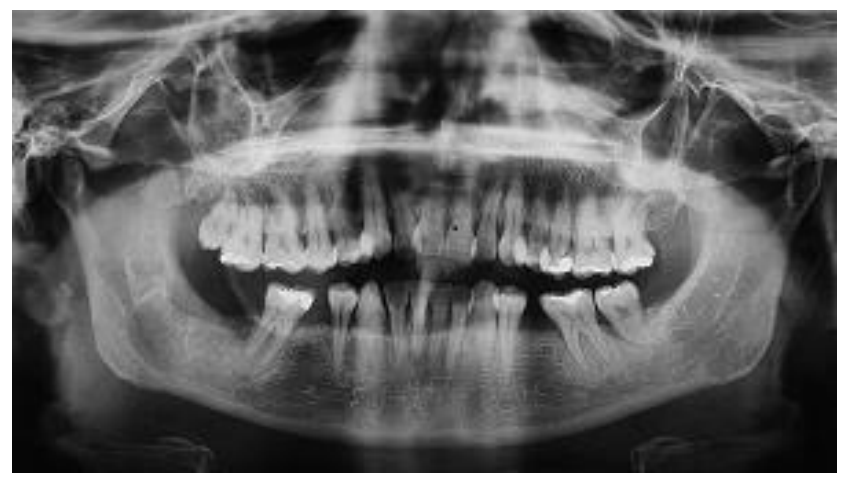

Figura 6: Exame radiográfico após 06 meses.

\section{DISCUSSÃO}

Segundo a literatura contemporânea o cisto odontogênico mais frequente nos ossos gnáticos é o cisto dentígero, normalmente ocorrem na segunda e terceira década de vida ${ }^{2-7}$. Clinicamente os cistos dentígeros são, usualmente, assintomáticos, com crescimento lento e caracterizados pelo envolvimento da coroa e erupção retardada da unidade dental envolvida $^{3,7,8}$. O caso clínico apresentado confirma os autores acima citados, pois ocorreu em uma paciente de 44 anos, em que a lesão estava associada a um terceiro molar inferior direito semi-incluso $\mathrm{e}$ impactado, sendo que esse dente normalmente apresenta-se incluso ou impactado.

Radiograficamente, o cisto dentígero é observado como uma imagem radiolúcida unilocular bem delimitada envolta por um halo esclerótico, associada à coroa de um dente incluso ${ }^{4-6,10,11}$. Os aspectos radiográficos apresentados nesse caso afirmam as características comuns citados anteriormente, em que a lesão é observada como uma imagem radiolúcida unilocular, bem delimitada envolta por um halo esclerótico, associada à coroa de um dente incluso.

Referente ao tratamento desse cisto existe algumas formas para serem realizados como, há descompressão, marsupialização e a enucleação. A escolha de tal procedimento é realizado de acordo com o tamanho do cisto, a idade do paciente, os dentes envolvidos e o envolvimento de outras estruturas anatômicas, alguns autores defendem que $o$ tratamento mais indicado na maioria dos casos, $85 \%$, é a enucleação ${ }^{20}$, com exodontia do dente não erupcionado, a fim de evitar recidiva da lesão $0^{21,22}$. A escolha pela enucleação do cisto, com extração dos elementos 47 e 48 durante a cirurgia, foi baseada na analise dos limites da lesão, através da tomografia computorizada, a posição do elemento 48 desfavorável. Relacionado com o resultado, discorremos a escolha do tratamento alcançado.

\section{CONCLUSÃO}

De acordo com o caso relatado, pode-se concluir que é fundamental um conhecimento aprofundado das características de tal lesão para um diagnóstico e uma técnica de tratamento sensato, utilizada para o cisto dentígero.

A supervisão do pós-operatório é primordial, pois devemos diligenciar exames radiográficos em intervalos regulares a fim de comprovar qualquer sinal de recidiva da lesão, concluir a recuperação do paciente para o desenvolvimento de atividades diárias e o seu convívio psicossocial.

\section{REFERÊNCIAS}

1. Jones TA, Perry RJ, Wake MJ. Marsupialization of a large unilateral mandibular dentigerous cyst in a 6-year-old boy - a case report. Dent Update. 
2003;30(10):557-61.

2. Chapelle KOM, Stoelinga PJ, de Wilde PC, Brouns JJ, Voorsmit RA. Rational approach to diagnosis and treatment of ameloblastomas and odontogenic keratocists. $\mathrm{Br} \mathrm{J}$ Oral Maxilofac Surg. 2004;42(5):381-90.

3. Sampaio RK, Prado R. Cirurgia dos cistos odontogênicos. In: Prado R, Salim M. Cirurgia bucomaxilofacial: diagnóstico e tratamento. Belo Horizonte: Medsi; 2004. p. 365-407.

4. Ustuner E, Fitoz S, Atasoy C, Erden I, Akyar S. Bilateral maxillary dentigerous cysts: a case report. Oral Surg Oral Med Oral Pathol Oral Radiol Endod. 2003; 95(5):632-35.

5. Tsukamoto G, Sasaki A, Akyama T, Ishikawa T, Kishimoto K, Nishiyama A et al. A radiologic analysis of dentigerous cyst and odontogenic keratocysts associated with a mandibular third molar. Oral Surg Oral Med Oral Pathol Oral Radiol Endod. 2001;91(6):743-47.

6. Daley TD, Wysocki GP. The small dentigerous cyst. A diagnostic dilemma.Oral Surg Oral Med Oral Pathol Oral Radiol Endod. 1995;79(1):77-81.

7. Regezi JA, Sciubba JJ. Patologia bucal: correlações clinicopatológicas. Rio de Janeiro: Guanabara Koogan; 2000.

8. Shafer WG, Hine MK, Levy BM. Tratado de patologia bucal. 4. ed. Rio de Janeiro: Guanabara Koogan; 1987.

9. Aziz SR, Pulse C, Dourmas MA, Roser SM. et al. Inferior alveolar nerve paresthesia associated with a mandibular dentigerous cyst. J Oral Maxillofac Surg. 2002;60(4):457-59.

10. Hyomoto M, Kawakami M, Inoue M, Kirita T. Clinical conditions for eruption of maxillary canines and mandibular premolars associated with dentigerous cysts. Am J Orthod Dentofac Orthop. 2003;124(5):515-20.

11. Thosaporn W, Iamaroon A, Pongsiriwet S, Ng $\mathrm{KH}$. A comparative study of epithelial cell proliferation between the odontogênic keratocyst, orthokeratinized odontogenic cyst, dentigerous cyst, and ameloblastoma. Oral Dis. 2004;10(1):22-6.

12.Neville BW, Damm DD, Allen CM, Bouquot JE. Patologia Oral \& Maxilofacial. 4.ed. Rio de Janeiro: Guanabara Koogan; 2016.

13.Bajaj MS, Mahindrakar A, Pushker N. Dentigerous cyst in the maxillary sinus: a rare cause of nasolacrimal obstruction. Orbit. 2003;22(4):289-92.

14.Kawamura JY, de Magalhães RP, Sousa SC, Magalhães MH. Management of a large dentigerous cyst occurring in a six-year-old boy. $\mathbf{J}$ Clin Pediatr Dent. 2004;28(4):355-57.

15. Motamedi MH, Talesh KT. Management of extensive dentigerous cysts. $\mathrm{Br}$ Dent $\mathrm{J}$. 2005;198(4):203-6.
16.Ertas U, Yavuz S. Interesting eruption of 4 teeth associated with a large dentigerous cyst in mandible by only marsupialization. J Oral Maxilofac Surg. 2003;61(6):728-32.

17.Kim SG, Yang BE, OH SH, Min SK, Hong SP, Choi JY. The differential expression pattern of BMP-4 between the dentigerous cystand the odontogenic keratocyst. J Oral Pathol Med. 2005;34(3):178-83.

18.Benn A, Altine M. Dentigerous cyst of infl amatory origin: a cliniopathologic study. Oral Surg Oral Med Oral Pathol Oral Radiol Endod. 1996;81(2):203-9.

19.Dunsche A, Babendererde O, Luttges J, Springer IN. Dentigerous cyst versus unicystic ameloblastoma - differential diagnosis in routine histology. J Oral Pathol Med. 2003; 32(8):486-91.

20.Fortin T, Coudert JL, Francois B, Huet A, Niogret F,Jourlin $\mathrm{M}$ et al. Marsupialization of dentigerous cyst associated with foreign body using 3D CT images: a case report. J Clin Pediatr Dent. 1997; 22(1):29-33.

21.Martínez-Pérez D, Varela-Morales $\quad M$. Conservative treatmentof dentigerous cysts in children: report of four cases. J Oral Maxillofac Surg. 2001;59(3):331-34.

22.Vaz LGM, Rodrigues MTV, Ferreira Júnior O. Cisto dentígero: características clínicas, radiográficas e critérios para o plano de tratamento. RGO. 2010;58(1):127-30.

\section{CONFLITO DE INTERESSES}

Os autores declaram não haver conflitos de interesse.

\section{AUTOR PARA CORRESPONDENCIA}

\section{Luciana Estevam Simonato}

luciana.simonato@universidadebrasil.edu.br

Submetido em 19/04/2018

Aceito em 27/06/2018 